\title{
El SEgURO PARA LOS SUBCAMPEONES ELECTORALES De la Alianza en 2009*
}

\author{
Insurance for good losers in the Alianza \\ in Legislative Elections in Chile, 2009
}

\section{PATRICIO NAVIA \\ Universidad Diego Portales}

\section{STEFFAN STERNBERG}

Universidad Diego Portales

\begin{abstract}
RESUMEN
En Chile, la dificultad de conseguir doblajes en el sistema binominal crea motivaciones contrapuestas entre partidos políticos que buscan maximizar la consecución de escaños, y candidatos que buscan ganar. Carey y Siavelis (2003) plantearon que la Concertación entregaba premios a los subcampeones que contribuían con un buen desempeño a la votación de su coalición. Aplicamos esa explicación a 2009, cuando la Alianza era favorita y los premios de consuelo serían más probablemente distribuidos entre sus subcampeones. Despejamos dudas sobre las causas que explican los duplos fuertes en la coalición con más probabilidades de ganar. Al mostrar que hay más duplos fuertes en la Alianza en 2009 que en elecciones anteriores apoyamos la tesis de los premios a los subcampeones.
\end{abstract}

Palabras clave: incentivos electorales, sistema electoral binominal, seguros para subcampeones, puestos de confianza en el Gobierno

\begin{abstract}
In Chile, the difficulty of winning both district seats under the binominal electoral system has generated opposite motivations for office-seeking political parties, which want to maximize their seat share, and individual candidates who want to win. Carey and Siavelis (2003) argue that the Concertación rewarded good losers who contributed to its overall vote share. We apply that explanation to the 2009 election, when the right-wing Alianza coalition was ahead and, therefore, any future rewards would be most likely to be distributed among its good losers. Our contribution clarifies doubts about the causes of strong legislative tickets at the district level in the coalition that would most likely win. In showing that the Alianza had stronger tickets in 2009 than in previous elections, we support the thesis of good losers' insurance.
\end{abstract}

Key words: electoral incentives, binominal electoral system, insurance for good losers, pre-sidential appointments

\footnotetext{
Agradecemos a nuestros colegas del Observatorio Político Electoral de la Universidad Diego Portales (OBPE-UDP) por sus comentarios, a Peter Siavelis y a los dos revisores anónimos por sus pertinentes y constructivas sugerencias. Este artículo fue parcialmente financiado por los proyectos Fondecyt Regular 1171051 y Conicyt/Fondap/15130009. Los errores u omisiones son de nuestra responsabilidad. La base de datos que hemos construido para el análisis estadístico se levantó con información públicamente disponible, pero estaremos gustosos de compartirla con cualquier persona que quiera replicar nuestros resultados.
} 


\section{INTRODUCCIÓN}

En Chile, las dinámicas del sistema electoral binominal, con listas conformadas por duplos en cada distrito, inducen a los partidos a promover la participación de candidatos en elecciones en las que tienen pocas posibilidades de ganar. La dificultad que plantea conseguir doblajes en el sistema binominal crea motivaciones contrapuestas en los partidos políticos que quieren maximizar la consecución de escaños, y los candidatos que aspiran a ganarlo.

Carey y Siavelis (2003:11-12) identifican la existencia de incentivos en forma de seguro para los candidatos legislativos que obtenían el segundo lugar (subcampeones) en la lista de la centro-izquierdista Concertación entre 1989 y 1997. Bajo los gobiernos de la Concertación parece haberse desarrollado un sistema informal que incentivaba la formación de listas con duplos de candidatos fuertes al ofrecer a los subcampeones cargos de gobierno después de la elección. Si bien este no fue un acuerdo formal, Carey y Siavelis muestran empíricamente su existencia. Al tener duplos fuertes, la Concertación optimizaba su posibilidad de asegurar una mayoría de los escaños electos en el Congreso. Los cargos de exclusiva confianza del presidente eran entregados a aquellos subcampeones de la Concertación que tuvieron un buen desempeño respecto a su compañero de lista, que contribuyeron a un alto desempeño comparativo respecto de la Alianza, y en mayor medida a quienes compitieron por escaños senatoriales (Carey y Siavelis 2003:13-14).

Presumiblemente, la condición dominante de la Concertación en 2005 mantuvo esa estructura de incentivos. Pero en 2009, después de 20 años de gobierno ininterrumpidos de la Concertación, la victoria del candidato de la Alianza, el RN Sebastián Piñera, produjo alternancia en el poder. En las elecciones legislativas también terminó la dominancia de la Concertación, cuando de los 120 escaños en la Cámara de Diputados, 58 fueron a la Alianza y 57 a la Concertación.

En 2009, la incertidumbre sobre el resultado de la elección hizo que aspirantes de la Alianza, en caso de perder la elección, pero sumando un alto porcentaje de votos a su lista, pudieran aspirar a un cargo de exclusiva confianza del Ejecutivo si Piñera ganaba la elección. La ventaja de Piñera en las encuestas incentivó a la coalición de derecha a presentar duplos fuertes en las elecciones legislativas, y a sus candidatos a correr el riesgo de participar con el fin de mejorar la votación de su coalición. Después que los seguros para subcampeones funcionaron bien para la Concertación en su intención de mantener unida a la coalición, el mecanismo parece haber sido ampliamente utilizado después de las elecciones de 2009. En su gobierno, el presidente Piñera nombró en cargos de confianza a 43 de los 63 candidatos subcampeones de la Alianza.

Los candidatos aliancistas en la circunscripción Quinta Costa fueron el UDI Joaquín Lavín y el RN Francisco Chahuán. Como en la mayoría de las unidades electorales, la Concertación y la Alianza se dividieron los escaños. Chahuán ganó el escaño de su lista al superar a Lavín por 2.241 votos (0,58\%). Lavín, exitoso ex 
alcalde de Las Condes (1992-1999), y quien había sido estrechamente derrotado en la elección presidencial de 1999 con la votación más alta de la Alianza hasta entonces, era ampliamente considerado como un candidato fuerte. Aunque resultaba improbable que la Alianza doblara la votación de la Concertación en esa circunscripción, la presencia de candidatos RN y UDI fuertes mejoraría la votación de esa coalición. La condición de subcampeón de Lavín y su aporte a la votación de la lista lo hizo acreedor de un premio de consuelo (o seguro), siendo nombrado ministro de educación en el primer gabinete de Piñera.

Ena Von Baer fue candidata independiente (pro-UDI) por la Alianza en la circunscripción Araucanía Sur. Si bien no salió electa, su 22,2\% ayudó a que su compañero de lista José García Ruminot (RN) lograra el escaño. Tras recibir apenas 742 votos $(0,27 \%)$ menos que García Ruminot, Von Baer fue nombrada Ministra Secretaria General de Gobierno en el primer gabinete de Piñera.

Por último, el diputado UDI Rodrigo Álvarez Zenteno, expresidente de la Cámara, se cambió al distrito 21 (Nuñoa y Providencia) en 2009 y buscó un escaño en la lista de la Alianza. Si bien la candidata RN Marcela Sabat ganó el cupo de la Alianza con un 27,4\%, el aporte de Álvarez (26,4\%) permitió a la Alianza obtener una mayoría de $53,8 \%$ en ese distrito. Álvarez fue nombrado Subsecretario de Hacienda en el gobierno de Piñera.

Ya que en ninguno de los casos hubo posibilidad de doblaje aliancista, la competencia fue intra-coalición. Lavín enfrentaba a un diputado de esa circunscripción, Von Baer se midió ante un senador incumbente y Álvarez enfrentó a la hija de un poderoso alcalde de una de las dos comunas del distrito. El riesgo que corrieron estos tres candidatos implicaba altos costos personales, pero ellos también sabían que existían altas posibilidades de que Piñera ganara la elección presidencial. Los tres fueron recompensados con importantes puestos de confianza del Ejecutivo por haber hecho el sacrificio de presentarse infructuosamente como candidatos y haber conseguido una alta votación que ayudó a superar la votación global de la Concertación.

Carey y Siavelis (2003) identificaron una serie de variables que explicaban la forma en que la Concertación entregaba premios a los subcampeones que competían en una elección y contribuían con un buen desempeño a la votación acumulada de su coalición. Nosotros aplicamos esas variables a la elección de 2009, cuando la Alianza aparecía como favorita para ganar la elección presidencial, y los premios de consuelo serían más probablemente distribuidos entre sus subcampeones.

Además de evaluar la validez de un citado y discutido mecanismo de incentivos para los candidatos en Chile, nuestra contribución permite despejar dudas sobre las causas que explican la presencia de duplos fuertes en la coalición con más probabilidades de ganar la elección presidencial. La primera alternancia en el Ejecutivo chileno tras la vuelta a la democracia ofrece un caso de estudio único, a la vez que también permite desechar las teorías alternativas formuladas 
para dar cuenta de la existencia de duplos fuertes en la Concertación. Si Carey y Siavelis (2003) están en lo correcto, debiéramos ver mayor presencia de duplos fuertes en la Alianza en 2009 que en elecciones anteriores. Además, como la Alianza está formada por dos partidos dominantes, frente a los cuatro que componen la Concertación y que solo se identifica ideológicamente con la derecha, la presencia de duplos aliancistas fuertes en 2009 no es atribuible al número de partidos o a la amplitud de su base de apoyo electoral. Finalmente, como la Alianza no estaba en el poder antes de 2009, la presencia de duplos fuertes en esa coalición no se podría asociar con el concepto de comisión de servicio - cuando un funcionario de gobierno es destinado a una labor especial, por ejemplo, ser candidato a diputado- discutido por Garrido (2009).

\section{DISCUSIÓN TEÓRICA}

¿Qué motiva a potenciales candidatos a competir en escenarios adversos? Las leyes electorales y los mecanismos de selección de candidatos pueden presentar barreras para quienes buscan alcanzar un escaño. Además, las motivaciones para la competencia difieren fuertemente según la información y las expectativas de éxito futuro, haciendo el contraste de oportunidades según quien es el candidato y contra quién compite. Así, la formación de carreras políticas y la ambición de los candidatos también influyen en la decisión de presentarse a una elección compleja. Las percepciones de los candidatos, partidos y coaliciones sobre el contexto político afectan la estructuración de las listas de candidatos de las coaliciones. La estructura de incentivos que enfrentan candidatos en escenarios electorales adversos también influye sobre la decisión de ser aspirante. De hecho, el contexto político a menudo presenta incentivos contrapuestos a candidatos que buscan ser electos y a coaliciones que buscan presentar duplos de candidatos fuertes.

\section{El rol de los sistemas electorales en las motivaciones de los candidatos}

Los sistemas electorales moldean la forma en que se produce la competencia entre candidatos y los esfuerzos de coordinación (Cox 1999). Los sistemas mayoritarios incentivan la formación de coaliciones de partidos que puedan obtener mejores rendimientos electorales por miedo a transformarse en perdedores absolutos. A su vez, desincentivan la competencia de candidatos menores que no cuenten con suficiente apoyo (Nohlen 2003, 2004; Reynolds et al. 2006). Por otro lado, los sistemas de representación proporcional harían más fácil a cada partido (o candidato independiente) competir por su cuenta dado sus bajos umbrales para conseguir un escaño.

La estructura de la papeleta también influye sobre la competencia (Reynolds et al. 2006:5; Von Baer 2009:179). Las listas abiertas incentivarían personalismos de candidatos y relativizarían la necesidad de buenas relaciones con el partido, 
aumentando la formación de facciones intra-partidarias, entre partidos y la competencia de "notables" (Reynolds et al. 2006:6). Las listas cerradas, donde los electores votan por etiquetas partidarias y sus cúpulas asignan el orden de los candidatos, incentivan la fidelidad y buena relación con sus líderes y el partido (Reynolds et al. 2006; Carey 2009:237). Este tipo de listas favorecería la candidatura de aspirantes desconocidos, pero de buen cometido al interior del partido. Luego, el conocimiento previo de las reglas del juego adecua la conducta de los candidatos, especialmente de los más débiles (Edwards 2009).

En Chile, la nominación de candidatos y la asignación de cargos en el Ejecutivo están determinadas por las dinámicas de competencia del sistema electoral. El sistema binominal, sindicado como el menos proporcional dentro de los sistemas proporcionales, fue creado en 1989 para disminuir el número de partidos políticos que existían antes de 1973 y evitar mayorías incontrarrestables en el Congreso (Navia 2005). Como el binominal distribuye dos escaños proporcionalmente entre las listas para cada uno de los 60 distritos en la Cámara de Diputados y las 19 circunscripciones senatoriales — con la fórmula d'Hondt-, el sistema tiende a favorecer a las dos coaliciones con mayor votación. Además de excluir a las listas menos votadas, el binominal dificulta que la coalición más votada transforme su mayoría electoral en una mayoría de escaños. Así, el binominal indujo a la institucionalización de un empate ficticio producto de la subrepresentación de partidos políticos pequeños y la sobre-representación de las coaliciones mayoritarias, especialmente la que tenía la segunda mayor votación.

Si bien el sistema binominal es proporcional, sus efectos mayoritarios presentan altas barreras de entrada a los candidatos aspirantes y desafiantes. De hecho, la asignación de dos escaños por distrito provoca que los partidos formen coaliciones y se dividan los cupos en los distintos distritos. De no existir coaliciones $-\mathrm{y}$ presentarse cada partido en forma individual a la elección- el sistema podría resultar siendo muy poco proporcional. Al requerir un 33,3\%+1 de los votos para asegurar un escaño y un $66,6 \%+1$ para garantizar ambos, los candidatos menos conocidos tienen menos incentivos para competir contra un candidato incumbente o fuerte en el distrito.

Al tener listas abiertas, el sistema binominal incentiva la presencia de candidatos conocidos con apoyo electoral más que partidario. De la misma forma, los candidatos que completan el duplo en las listas legislativas tendrán menos posibilidades de resultar electos cuando acompañan a candidatos fuertes. En el caso de que ambos candidatos sean considerados fuertes, las listas abiertas inducirán a una mayor competencia intra-coalición. Además, los incentivos del binominal dan una ventaja inicial a incumbentes y candidatos fuertes, ya que los votos para los desafiantes aumentan tanto las preferencias totales de la lista como las chances de elección de su compañero de duplo.

Por último, una magnitud de distrito de dos escaños desincentivará la participación de aspirantes que conciten bajo apoyo. La baja cantidad de escaños a repartir, con altas barreras de entrada y listas abiertas, disminuye las 
expectativas de éxito para los aspirantes. Incluso cuando hay dos candidatos fuertes en una coalición, la probabilidad de que uno quede fuera es alta.

Las dinámicas de competencia en el sistema binominal presentan dificultades tanto para los aspirantes como para los duplos de candidatos fuertes. Luego, las motivaciones a participar que tienen aspirantes con pocas posibilidades de ganar, o un segundo candidato fuerte, deben estar relacionadas a otras variables, como los seguros para los subcampeones.

\section{Mecanismos de selección de candidatos}

El nivel de inclusividad del mecanismo de selección de candidatos puede determinar la competitividad de un distrito (Hazan y Rahat 2006: 371). También tiene efecto sobre el tipo de candidatos que son seleccionados y los clivajes societales que representan (Field y Siavelis 2008), generando tensión entre los incentivos opuestos de los partidos/coaliciones y los candidatos.

En Chile, la selección de candidatos y la conformación de las listas están casi exclusivamente en manos de los partidos (Navia 2008). Entre los mecanismos más exclusivos —ampliamente utilizados por los partidos, especialmente los de derecha - se encuentran la designación por cúpulas partidarias y comités centrales. Si bien permiten aumentar la disciplina de los militantes y congresistas que buscan ser designados, tienden a perjudican a candidatos menos conocidos de la colectividad. Por otro lado, los métodos inclusivos como las juntas provinciales y primarias de todo tipo (abiertas, cerradas y subtipos), incentivan una mayor rotación parlamentaria y alienta a los aspirantes a competir (Toro y García 2008: 397). Aunque los métodos inclusivos fueron escasamente utilizados por algunos partidos de la Concertación, su uso se limitó a la propuesta de candidatos por sub-comités regionales y agrupaciones partidarias condicionales a la aprobación de sus cúpulas (Field y Siavelis 2011: 804).

Como el sistema binominal dificulta que una lista obtenga los dos escaños, es altamente probable que uno de los dos integrantes de la lista resulte derrotado. Tanto para optimizar la posibilidad de doblar como para evitar ser doblados, los partidos buscan presentar duplas de candidatos lo suficientemente competitivas. Esto se consigue con dos candidatos fuertes que aspiren a conseguir ambos escaños, o bien con un candidato fuerte acompañado de uno más débil para superar el umbral mínimo que requiere un escaño. A su vez, los candidatos que razonablemente aspiran a ganar un escaño prefieren compañeros de lista más débiles que ayuden a conseguir el umbral mínimo de votación para ganarlo, pero que no constituyan una amenaza. Por lo tanto, los incentivos para las coaliciones y los candidatos son contrapuestos.

Dado que las coaliciones son multipartidistas, el sistema binominal obliga a negociaciones entre partidos para constituir las listas de candidatos. Según Siavelis (2005), el binominalismo hace convivir tres lógicas de negociación en la 
selección de candidatos: la selección a nivel de partidos, las negociaciones entre subpactos, y la formulación de listas de las coaliciones.

Un incumbente tendría más oportunidades de tener el apoyo de la coalición en la competencia que un desafiante, quien tendría que salir airoso en cada una de las lógicas de negociación para recibir apoyo. La negociación en duplos de candidatos se volvería más riesgosa cuando se intenta desafiar al incumbente, o a quien tiene la ventaja inicial en los tres niveles de negociación. Por lo mismo, los costos para los desafiantes aumentan y las oportunidades de ganar disminuyen. Así, resulta clave entender los incentivos que tienen esos candidatos para competir.

El método de selección de candidatos no es la única variable que influye en la fortaleza de los duplos. También lo hace el tamaño y la composición de los distritos (Freidenberg 2003; Alcántara y Freidenberg 2009). Otros determinantes de la competitividad de las listas son las mayorías legislativas que tengan los partidos en esas localidades (Siavelis y Morgenstern 2008; Alcántara y Freidenberg 2009), la condición de oficialismo u oposición de los competidores (De Luca et al. 2002; Alcántara y Freidenberg 2009), los resultados electorales anteriores para el cargo en disputa y los resultados en otros niveles institucionales de gobierno (Alarcón y Freidenberg 2007). Pero la selección de candidatos, especialmente en un contexto de magnitud de distrito baja y coaliciones multipartidistas, es una de las variables relevantes para determinar la fortaleza de una lista. Luego, la selección de candidatos expone la contradicción entre partidos/coaliciones que aspiran a presentar duplos fuertes y candidatos que prefieren compañeros de lista débiles. Para destrabar esa contradicción, los partidos deben recurrir a incentivos adicionales, tales como los seguros para subcampeones.

\section{Carreras políticas y la ambición de los candidatos}

Carey y Shugart (1995) argumentan que los candidatos pueden comportarse de distintas maneras según las variables institucionales que enfrenten. Estas son el grado de control que tienen los líderes de las colectividades al acceso a cupos de candidatos, la estructura de la papeleta (tipo de lista y repartición por candidato, partido o lista), la estructura del voto mismo (voto único o múltiple) y la magnitud de distrito. Además, si bien las elecciones son un proceso que involucra incertidumbre, ya que en teoría supone la posibilidad de perder, los candidatos incumbentes suelen tener ventaja por sobre los aspirantes (Groseclose 2001).

Según la teoría de acción racional, los políticos que buscan competir en elecciones realizan un cálculo de costo y beneficio al participar (Downs 1957). Además, toman en cuenta factores como la información existente y las expectativas de éxito futuro (Black 1972; Alcorta 2011). De hecho, una causa que podría explicar la participación de candidatos con un cálculo racional desalentador es la posibilidad de construir carreras políticas. Diermeier et al. (2002) señalan que 
los políticos toman decisiones de futuro sobre sus carreras según su ambición estática (ir por la reelección), ambición progresiva (participar por escaños de mayor rango) o dejar la carrera política. Los aspirantes o desafiantes aplican la misma lógica de costos y beneficios en su ambición por mayores utilidades al buscar un escaño parlamentario.

En el caso chileno, las variables institucionales configuran la posibilidad de escoger un solo candidato en listas abiertas, la primacía de los partidos políticos en la selección de candidatos, y una magnitud de distrito pequeña. También se aprecian altas barreras de entrada por la repartición proporcional de escaños asociada a la baja magnitud de distrito. Además, la alta tasa de re-elección de los incumbentes dificulta el camino para nuevos candidatos (Bunker y Navia 2009). De hecho, tanto en la Concertación como en la Alianza, más de la mitad de los diputados electos en 2009 eran incumbentes (Munita y Gillmore 2009).

La mezcla de listas abiertas, la selección de candidatos dominada por líderes de partidos, la pequeña magnitud de distrito y los altos umbrales de entrada para conseguir escaños desincentivarían fuertemente a candidatos aspirantes o desafiantes. Aun así, aquellos postulantes que tienen aspiraciones de construir una carrera política podrían verse inducidos a participar cuando existe la posibilidad de recibir un seguro para subcampeones.

\section{Los premios para los subcampeones}

Carey y Siavelis presentan un modelo de regresión logística para explicar la probabilidad de asignación de cargos de exclusiva confianza a los subcampeones de la Concertación entre 1989 y 1997. Su modelo incluye la cámara a la que postulaban los subcampeones, la razón de votación entre las coaliciones (la votación concertacionista dividida por la votación de la Alianza) y al interior de la lista de la Concertación (la votación del candidato más votado divida por la votación del candidato con menos votos).

Nuestra variable dependiente es dicotómica — “asignación de cargo" - y discrimina entre aquellos candidatos que fueron nombrados a un cargo de confianza del Ejecutivo en el periodo legislativo inmediatamente posterior y los que no recibieron cargo. Para denominarse subcampeón, el candidato debe cumplir las siguientes condiciones:

- compitió en duplos, de forma que la lista no podría haber presentado un solo candidato en el distrito,

- uno de los participantes de la lista resultó electo, transformándose su compañero en un subcampeón, y

- no se ganó ambos escaños del distrito (doblaje), ya que en tal caso no habría un perdedor en la lista. 
Carey y Siavelis muestran que los subcampeones de listas concertacionistas que obtuvieron una razón Coalición mayor a 1,8 —o el 90\% de los votos necesarios para doblar a la Alianza - se agruparon como más probables, mientras que aquellos con una razón Coalición entre 1,0 y 0,5 -igual o menor votación que la lista derechista - fueron clasificados como menos probables. La Figura 1 muestra las variables que se incluyen en el modelo de Carey y Siavelis (2003).

Figura 1. Modelo regresión Carey y Siavelis (2003)

\begin{aligned} & \hline $\operatorname{Pr}($ Asignación de Cargo $)= \mathrm{A}($ Constante $) \\ &$\hline$+\mathrm{b} 1$ (Cámara) \\ &$+\mathrm{b} 2$ (Razón Coalición) \\ &$+\mathrm{b} 3$ (Razón Concertación) \\ & \hline\end{aligned}

Fuente: adaptación de Carey y Siavelis (2003: 8)

En respuesta a Carey y Siavelis, Garrido y Navia (2005) proponen dos argumentos alternativos para explicar la presencia de duplos fuertes en la Concertación. Explican que la coalición de centro-izquierda representaría dos de los tres tercios históricos de la política chilena y señalan que este pacto está conformado por un mayor número de partidos políticos que la Alianza, por lo que es obvia la inclusión de más candidatos fuertes (Garrido y Navia 2005: 2). Aun así, reconocen que no logran una confianza estadística ni metodológica superior al argumento de Carey y Siavelis (Garrido y Navia 2005: 2).

En una respuesta adicional posterior, Garrido (2009) plantea que los duplos fuertes de la Concertación se explican por la presencia de políticos que compiten como candidatos en comisión de servicio, y que después de no ganar un escaño vuelven a puestos de confianza en el gobierno. Los candidatos de la Concertación serían funcionarios públicos que se refugian en el aparato estatal durante el periodo de gobierno, para luego dejar sus puestos y competir en comicios parlamentarios (Garrido, 2009: 112).

Al reproducir el modelo de Carey y Siavelis, pero aplicado a la Alianza en 2009, podemos evaluar también las hipótesis de Garrido y Navia (2005) y Garrido (2009). Si los duplos fuertes de la Concertación fueron resultado de que esa coalición congregaba partidos de centro e izquierda o fueron producto de candidatos que se presentaban en comisión de servicio - y no se constituían por los posibles premios de consuelo-, en 2009 la Concertación nuevamente debió tener duplos fuertes, no así la Alianza.

Si bien utilizamos el modelo base de Carey y Siavelis, también integramos otras variables de control en distintas etapas, ya sea para buscar una mayor confianza estadística o para analizar otras variables que podrían explicar la asignación de cargos a los subcampeones de la Alianza. Incorporamos el partido al que 
pertenecen los subcampeones (UDI, RN, Chile Primero - $\mathrm{O}$ independientes en la lista Alianza - IND), el aporte de cada subcampeón a la votación del partido (su votación como porcentaje de la votación nacional del partido), el gasto electoral del subcampeón (comparación del gasto del subcampeón del distrito en 2005 y 2009 en pesos ajustados de 2009) y el sexo del candidato.

Trabajamos con regresiones logísticas binarias que permiten analizar variables dicotómicas —en este caso, asignación de cargo $(0=\mathrm{Si} ; 1=\mathrm{No})$. Así, testeamos las distintas variables en un continuo del tipo "condiciones más favorables" para conseguir un cargo. Además, la significancia de los modelos de regresión binaria permite saber si la variable influye en las condiciones del subcampeón para que se le asigne un cargo de confianza del Ejecutivo.

A partir de las contribuciones de Carey y Siavelis, articulamos dos hipótesis.

H1: Las mayores opciones de ganar la elección presidencial inducen la formación de duplos más fuertes en la Alianza en 2009 que en años anteriores.

H2: Siguiendo a Carey y Siavelis, la probabilidad de asignación de cargos de confianza del Ejecutivo depende del tipo de escaño que aspiraba a conseguir el subcampeón, del desempeño electoral intra-coalición del subcampeón, y de su desempeño respecto de la lista de la Concertación.

Las razones para creer que el tipo de premio recibido tiene relación con estas variables independientes ameritan explicación. El tipo de cargo al que se aspira, Senador o Diputado, influye sobre los cargos de confianza en el gobierno a los que pudieran acceder los subcampeones. Si bien ambas cámaras tienen poderes formales similares, hay menos senadores que diputados, por lo que el cargo de Senador es más preciado (Carey y Siavelis 2003: 13). Por eso, el perfil de un candidato al Senado es más idóneo para cargos como Ministro, Subsecretario, Embajador, Intendente o Gobernador. Asignamos valor " 1 " a los candidatos a diputados que fueron subcampeones y valor " 2 " a los subcampeones aspirantes al Senado.

La competencia al interior de la lista (intra-coalición) resulta de dividir la cantidad de votos del candidato que obtiene la primera mayoría y de su compañero de lista. A mayor competencia, mayores deberían ser las posibilidades de recibir atractivos cargos en el gobierno, dado que los duplos fuertes tienen más posibilidades de doblar la votación de la otra coalición o evitar ser doblados.

Ahora bien, pudiera ser que la Alianza tenga dos candidatos débiles, pero con votación similar. Esto produciría mucha competencia intra-coalición, pero no aumentará las posibilidades de doblar o incluso ganar un escaño. Por eso, evaluamos también la competencia entre coaliciones. En casi todos los casos, esto significa competencia Alianza/Concertación. La competencia entre coaliciones busca identificar distritos donde la Alianza era fuerte. Por eso, ignoramos los casos en que una tercera lista estuvo entre las dos más votadas en un distrito, pues buscamos identificar las lógicas de premios a subcampeones 
de coaliciones que pueden ganar la elección presidencial. Una mayor "razón Coalición" (división entre la votación de la lista con más votos y la segunda lista más votada) identifica más competencia por los escaños a repartir, ya sea asegurando un escaño o aspirando a conseguir ambos. El subcampeón de la Alianza en un distrito más competitivo es más importante para esa coalición que un subcampeón en un distrito menos competitivo.

Para los datos de premios de consuelo, hemos recopilado información públicamente disponible en sitios web del Gobierno, incluidos los sitios de transparencia activa que entregan información sobre personal a contrata y honorarios. Verificamos si los subcampeones de la Alianza ocuparon puestos de confianza del Ejecutivo en los cargos de Intendente, Gobernador, Ministro, Subsecretario, Seremi, Embajador y otros, en el periodo legislativo inmediatamente posterior a la elección (2010-2014). Como los seguros premian la competencia en elecciones inciertas con la confianza del Ejecutivo, aquellos subcampeones que llenan vacancias en el Congreso (atribución exclusiva de los partidos), no son contabilizados como acreedores de seguros.

\section{LOS DATOS}

El Cuadro 1 muestra los resultados de las elecciones presidenciales y legislativas para la Alianza y la Concertación desde 1989 a 2009. A partir de 1999, las elecciones presidenciales se decidieron por márgenes estrechos. Los resultados de 1999, 2005 (con la votación combinada de ambos candidatos derechistas) y 2009 muestran el aumento en las posibilidades de éxito de la Alianza y la creciente vulnerabilidad de la Concertación. Al ganar la elección presidencial en 2009, la Alianza pudo designar cargos de confianza en el Ejecutivo. 
Cuadro 1. Votación de Concertación y Alianza en elecciones en Chile, 1989-2009

\begin{tabular}{rrrrrrr}
\cline { 2 - 7 } Año & \multicolumn{3}{c}{ Concertación } & \multicolumn{4}{c}{ Alianza } \\
\cline { 2 - 7 } & $\begin{array}{c}\text { Presidencial } \\
\left(2^{\mathrm{a}} \text { vuelta) }\right.\end{array}$ & $\begin{array}{c}\text { Diputados } \\
\text { (\# escaños) }\end{array}$ & $\begin{array}{c}\text { Diferencia } \\
\text { Pres.-Dip. } \\
\%\end{array}$ & $\begin{array}{c}\text { Presidencial } \\
\left(2^{\mathrm{a}} \text { vuelta) }\right.\end{array}$ & $\begin{array}{c}\text { Diputados } \\
(\# \text { escaños })\end{array}$ & $\begin{array}{c}\text { Diferencia } \\
\text { Pres.-Dip. } \\
\%\end{array}$ \\
\hline 1989 & 55,2 & $51,5(69)$ & 3,7 & 29,4 & $34,2(48)$ & $-4,8$ \\
1993 & 58,0 & $55,4(70)$ & 2,6 & 24,4 & $36,7(50)$ & $-12,3$ \\
1997 & - & $50,5(69)$ & -- & -- & $36,3(47)$ & -- \\
1999 & $48,0(51,3)$ & -- & -- & $47,5(48,7)$ & -- & -- \\
2001 & - & $47,9(62)$ & -- & -- & $44,3(57)$ & -- \\
2005 & $46,0(53,5)$ & $51,8(65)$ & $-5,8$ & $48,6^{*}(46,5)$ & $38,7(54)$ & 9,9 \\
2009 & $29,6(48,4)$ & $44,4(57)$ & $-14,8$ & $44,1(51,6)$ & $43,5(58)$ & 0,6 \\
\hline
\end{tabular}

* Se suma la votación de los dos candidatos de la Alianza en primera vuelta

Fuente: elaboración propia con datos de www.elecciones.gob.cl

Para evaluar la fortaleza de los duplos de candidatos en la Alianza y Concertación, el Cuadro 2 muestra el promedio de la razón entre el candidato que sumó más votos en su lista y su compañero entre 1989 y 2009. Esta razón fluctúa entre un número apenas superior a uno (competencia perfecta) y cualquier número positivo superior. Mientras una menor razón supone duplos más fuertes, una menor desviación estándar indica mayor uniformidad en las competencias en cada coalición. La Concertación presenta duplos más fuertes que la Alianza en todas las elecciones hasta 2005. En ese año, la Alianza presentó duplos más fuertes que en cualquier elección anterior, pero aun así fueron en promedio más débiles que los de la Concertación. En 2009, la Alianza presentó duplos más fuertes que nunca antes y más fuertes que la Concertación.

La evidencia del Cuadro 2 es consistente con nuestra primera hipótesis. Cuando tuvo más posibilidades de ganar (cuando las elecciones presidenciales se anticipaban como más competitivas), la Alianza presentó duplos más fuertes. En 2005, cuando la votación combinada de los dos candidatos presidenciales aliancistas en primera vuelta superó la votación de la candidata de la Concertación, los duplos de la Alianza fueron también más fuertes que en cualquier elección anterior. En 2009, cuando el candidato de la Alianza era el favorito para ganar — desde antes que se inscribieran las listas de candidatos al Congreso-, la Alianza presentó duplos más fuertes que la Concertación.

Aunque la Alianza había logrado muy pocos doblajes en elecciones anteriores, en 2005 y 2009 el conglomerado presentó duplos más fuertes que antes. La presencia de dos candidatos presidenciales en la Alianza en 2005 - un RN y un UDI - indujeron al fortalecimiento de duplos, en tanto cada partido aspiró a potenciar a sus propios candidatos. En 2009, en cambio, las altas expectativas de victoria del candidato único de la Alianza indujeron a la formación de duplos más fuertes. 
Cuadro 2. Razón de primer y segundo lugar en cada coalición en elecciones legislativas, 1989-2009

\begin{tabular}{crrrr}
\hline & \multicolumn{4}{c}{ Promedio de razón primero/segundo de la lista } \\
\cline { 2 - 5 } Año & Alianza & $\begin{array}{c}\text { Desviación } \\
\text { Estándar }\end{array}$ & $\begin{array}{c}\text { Concerta- } \\
\text { ción }\end{array}$ & $\begin{array}{c}\text { Desviación } \\
\text { Estándar }\end{array}$ \\
\hline 1989 & 3,67 & 4,53 & 2,45 & 2,47 \\
1993 & 4,29 & 3,32 & 2,47 & 2,38 \\
1997 & 5,24 & 5,78 & 2,19 & 1,66 \\
2001 & 4,74 & 4,85 & 3,05 & 4,20 \\
2005 & 2,96 & 2,54 & 2,72 & 2,01 \\
2009 & 2,78 & 2,11 & 3,18 & 3,05 \\
$1989-2009$ & 3,95 & - & 2,68 & - \\
\hline
\end{tabular}

Fuente: elaboración propia con datos de Garrido (2009) y www.elecciones.gov.cl

La Figura 2 muestra la evolución en la variación promedio de la competitividad de los duplos de ambas coaliciones entre 1989 y 2009. La Concertación muestra más competitividad entre 1989 y 2001. Luego se produce una diferencia mínima de 0,24 a favor de la Concertación en 2005. Ya en 2009, la Alianza tiene duplos más competitivos que la Concertación.

En 1989 y 1993, años con elecciones presidenciales y legislativas concurrentes, la Concertación tuvo duplos más fuertes que la Alianza. Las altas expectativas de victoria de los candidatos presidenciales concertacionistas, presumiblemente contribuyeron a que se presentaran candidatos en distritos donde tenían pocas posibilidades de doblar con la expectativa de recibir premios de consuelo.

En 1997, cuando solo hubo elecciones legislativas y la presidencia estaba en manos de la Concertación, se produjo la mayor diferencia en la fortaleza de duplos entre ambas coaliciones. Había certeza que solo la Concertación podría entregar premios de consuelo. Lo mismo ocurrió en 2001, cuando recién se iniciaba el gobierno de Ricardo Lagos (2000-2006). La certeza de que el Ejecutivo seguiría en manos de la Concertación ayudó a que se presentaran más candidatos fuertes en esa coalición, sabiendo que habría acceso a premios de consuelo.

En las elecciones concurrentes de 2005, el escenario fue distinto. Con la mejora progresiva del desempeño electoral de la derecha, el ambiente de incertidumbre era mayor. Si cualquiera de los candidatos presidenciales de la Alianza, Lavín o Piñera, resultaba electo, los subcampeones de la Alianza podrían aspirar a premios de consuelo. Así, la fortaleza de los duplos legislativos aliancistas fue sustancialmente mayor en 2005 que en todas las elecciones anteriores.

Finalmente, en las elecciones concurrentes de 2009, la Alianza presentó duplos más competitivos que la Concertación. El temprano favoritismo en las encuestas de Piñera aparentemente indujo a más aspirantes de la Alianza a presentarse 
como candidatos al Congreso. A su vez, el pobre desempeño en las encuestas del candidato concertacionista Eduardo Frei disuadió a potenciales aspirantes de la Concertación a buscar un escaño.

Figura 2. Evolución de razón primer/segundo lugar en elecciones legislativas, 1989-2009

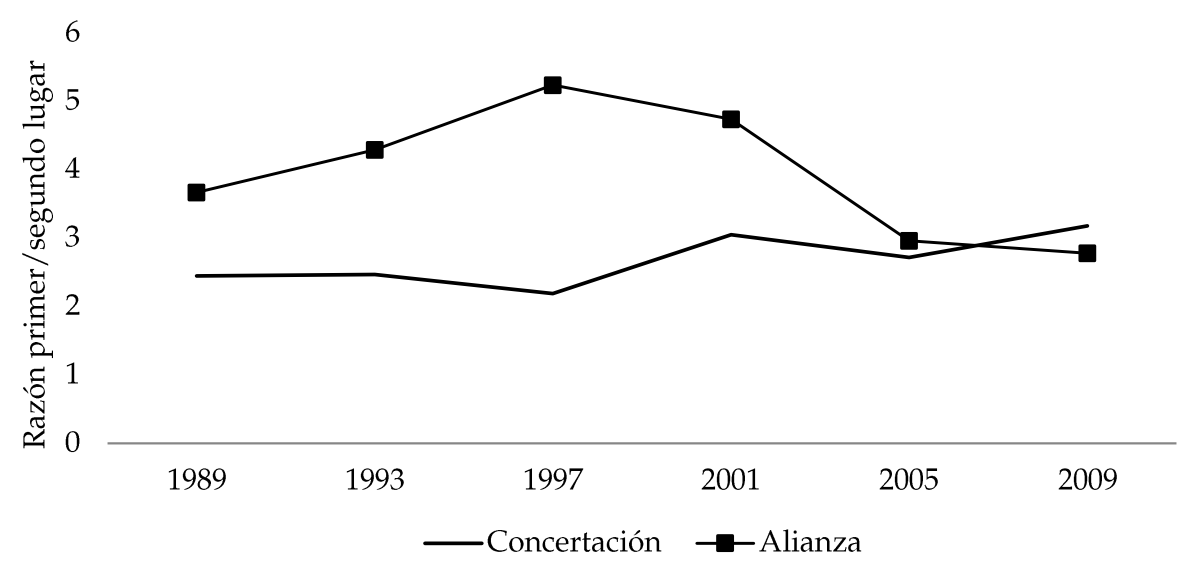

Fuente: elaboración propia con datos de Garrido (2009) y www.elecciones.gov.cl

El Cuadro 3 muestra los resultados de la competencia entre la Alianza y la Concertación y la distribución de escaños en la Cámara de Diputados y el Senado. Se presentan los doblajes conseguidos por la coalición derechista, los escaños ganados y el número de duplos. Si bien la Alianza consiguió un solo doblaje por elección entre 1993 y 2009 —en el distrito 23, el de más ingresos en el país- la Concertación bajó de 11 doblajes en la Cámara en 1989 a ninguno en 2009. En el Senado, la Concertación alcanzó su número más alto de doblajes en 1989, en tres circunscripciones.

Los datos del Cuadro 3 permiten asociar el declive de los duplos concertacionistas con los mejores resultados de la derecha en obtención de escaños desde 2001. La disminución en las probabilidades de doblajes concertacionistas alimentó las expectativas aliancistas de conseguir escaños para sus duplos. A su vez, el menor número de doblajes esperados en la Concertación pudo desincentivar a aspirantes de esa coalición a presentarse en distritos donde tenían pocas posibilidades de ganar. Si disminuía el número de distritos donde la Concertación escogía a sus dos candidatos, era razonable esperar que hubiera menos candidatos dispuestos a presentarse para terminar en segundo lugar (sabiendo que había pocas chances de que su coalición ganara la elección presidencial y ellos accedieran a premios de consuelo).

Entre 1989 y 2009, la Alianza ha presentado entre 59 y 60 duplos de candidatos en los 60 distritos del país. En el Senado, la Alianza ha presentado duplos en 
casi todas las elecciones, salvo en 2001, cuando el Ejecutivo estaba en manos de la Concertación y no había elecciones concurrentes. Ese año, la Alianza presentó duplos solo en la mitad de las circunscripciones que escogían senadores. Como la definición de subcampeón supone que el otro candidato de la coalición ganó un escaño, el número de subcampeones de la Alianza aumentó a medida que disminuyó el número de doblajes de la Concertación. Por eso, cuando la Concertación no alcanzó ningún doblaje en 2009, la Alianza tuvo 55 subcampeones en 60 distritos. En uno de los distritos restantes la Alianza ganó los dos escaños. En otro, presentó un solo candidato. En los otros tres no logró elegir a un diputado y, por lo tanto, no hubo subcampeón de la Alianza.

En el Senado, la Alianza presentó ocho duplos de candidatos en las nueve circunscripciones que renovaban escaños (Antonio Horvath fue candidato único de la Alianza en la circunscripción 18). La Alianza ganó ocho escaños y, por lo tanto, tuvo ocho subcampeones. En consecuencia, la suma de subcampeones de la Alianza en 2009 en ambas cámaras fue 63 -55 diputados y 8 senadores.

Cuadro 3. Distribución de escaños a nivel nacional en elecciones legislativas, 1989-2009

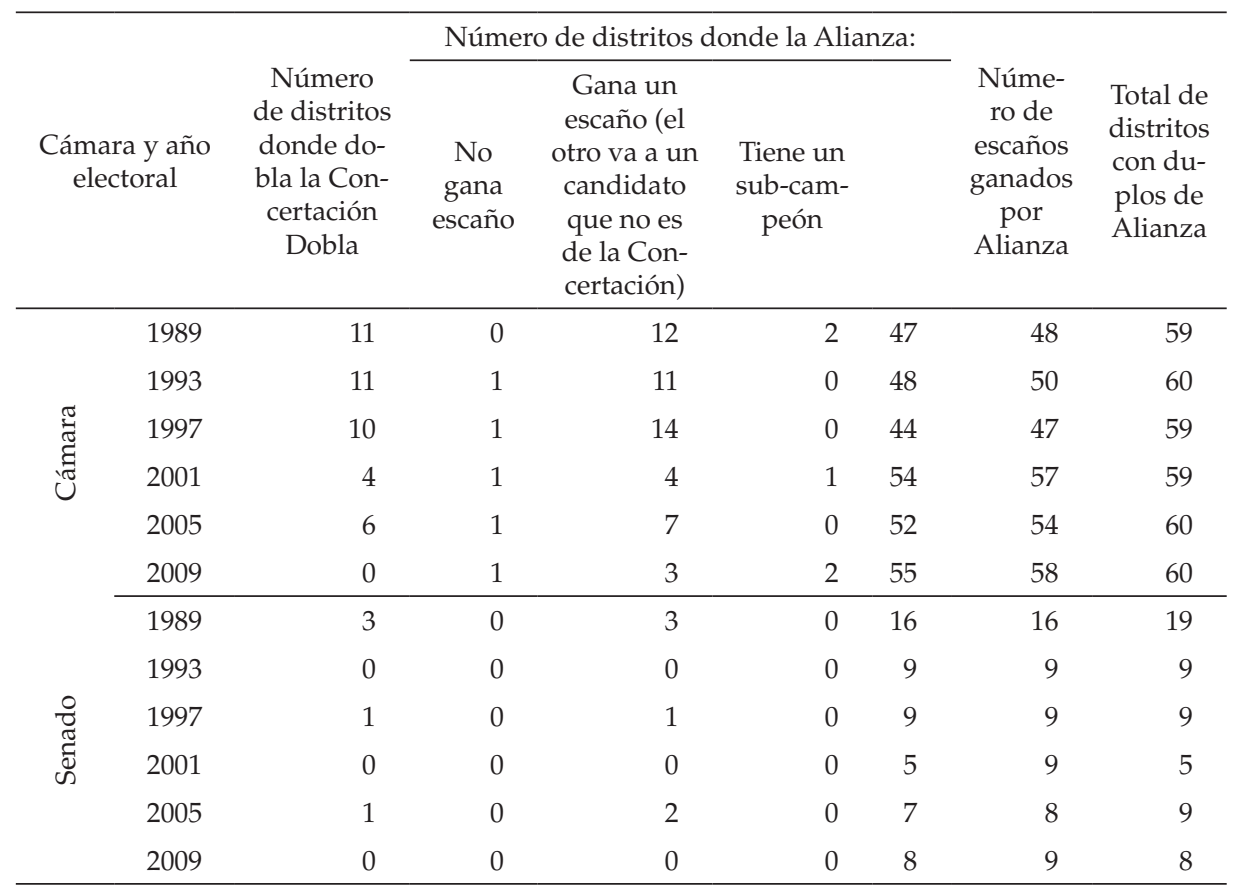

Fuente: elaboración propia en base a Garrido y Navia (2005) y datos de www.elecciones.gov.cl 
El Cuadro 4 muestra que, de los 63 subcampeones de la Alianza en 2009, 42 $(66,7 \%)$ obtuvieron un cargo de confianza del Ejecutivo en el periodo 20102014. Esta evidencia es consistente con lo señalado por Lobos et al. (2015), quienes aseveran, sin mostrar datos, la existencia de premios de consuelo. El Cuadro 4 también muestra la evolución en el número de subcampeones con cargos de confianza en cada año del gobierno de Piñera. La caída en 2012 en el número de candidatos que tenían premios de consuelo parece apoyar la tesis de Garrido (2009) que postula que los candidatos de cada coalición pueden ser funcionarios en comisión de servicio. Como en 2012 hubo elecciones municipales y en 2013 comicios legislativos, bien pudiera ser que algunos de los candidatos de la Alianza en 2009, que fueron beneficiados con un puesto durante la administración Piñera, hayan dejado sus cargos para volver a ser candidatos. Otra explicación es que, dada la baja aprobación presidencial y las altas expectativas que la Concertación volvería al poder en las elecciones de 2013, algunos funcionarios optaron por continuar sus carreras profesionales fuera del gobierno.

El Cuadro 4 también presenta el sueldo promedio de los excandidatos en sus cargos de confianza respectivos. El alto promedio de sueldos para subcampeones está relacionado con el tipo de cargos. Varios de los subcampeones ocuparon cargos de Ministros y Subsecretarios, puestos con los sueldos más altos del sector público.

Cuadro 4. Número de seguros de subcampeones y sueldo promedio, 2010-2014

\begin{tabular}{lrrrrrr}
\hline Subcampeones electorales & 2010 & 2011 & 2012 & 2013 & 2014 & $\begin{array}{c}\text { Total } \\
\text { segu- } \\
\text { ros }\end{array}$ \\
\hline Tienen cargo* & 35 & 40 & 36 & 28 & 19 & 42 \\
No tienen cargo & 27 & 21 & 24 & 32 & 41 & \\
\% que recibe cargo & 56,5 & 67,2 & 61,7 & 48,3 & 33,3 & 66,7 \\
$\begin{array}{l}\text { Sueldo promedio en \$ chilenos } \\
\text { (millones) }\end{array}$ & 3,9 & 3,9 & 4,4 & 4,3 & $* *$ & -- \\
$\begin{array}{l}\text { Sueldo promedio en US (tasa } \\
\text { de cambio abril de 2017) }\end{array}$ & US\$ & US\$ & US\$ & US\$ & $* *$ & -- \\
\# cargos con información de & 30 & 5.873 & 6.627 & 6.476 & & \\
sueldos & 34 & 30 & 25 & 9 & 30 \\
\hline
\end{tabular}

* No se encontró información sobre el tiempo que ejerció su cargo el subcampeón Mario Varela, candidato en el Distrito 18. Se excluye del conteo anual, pero no del total de seguros.

** Como el gobierno de Piñera terminó en marzo de 2014, excluimos los sueldos de los dos primeros meses de ese año.

Fuente: elaboración propia con datos de www.gobiernotransparentechile.cl

Adicionalmente, hemos encontrado información de ocho subcampeones de la Alianza en 2009 que fueron nombrados gerentes o directores en empresas privadas después de 2009. Ellos son Marcelo Forni, quien tomó la gerencia 
general de CorpArtes; Pablo Jofré, quien formó parte de Señalización y Publicidad Limitada; Christian Espejo, en Emres; Lautaro Alliende, quien volvió a la gerencia general de Smart Security; Claudio Eguiluz, quien formó parte del directorio de Enacar S.A.; y Mauricio Gejman, Harry Jurgensen y Cecilia Villouta, quienes se desempeñaron como empresarios independientes. Si bien la posibilidad de acceder a empleos en el sector privado con altas expectativas de sueldo podría inducir al subcampeón a no querer un puesto de confianza en el Ejecutivo, este análisis solo evalúa a aquellos subcampeones que accedieron a puestos de gobierno.

\section{MODELOS PREDICTIVOS DEL ACCESO A PREMIOS DE CONSUELO}

Al igual que Carey y Siavelis (2003), presentamos un modelo estadístico para dar cuenta de qué variables determinan el acceso a premios para los subcampeones. Si bien uno de nuestros modelos usa las mismas variables que Carey y Siavelis, agregamos otros que añaden variables adicionales que pudieran explicar las lógicas que determinan el acceso de subcampeones a cargos de confianza en el Ejecutivo.

El Modelo 1 en el Cuadro 5 es el propuesto originalmente por Carey y Siavelis (2003). Este modelo integra las variables Cámara a la que se postula, Razón Coalición y Razón Alianza (diferencia en el porcentaje de votos entre el primer y segundo lugar en cada coalición). La regresión binaria predice correctamente el $69,8 \%$ de los casos. Esto no es muy auspicioso, en tanto sabemos que el 66,7\% de los subcampeones recibió un cargo de confianza. Bastaría con afirmar que todos los subcampeones recibirán un cargo para tener una capacidad predictiva del 66,7\%. Luego, el modelo original de Carey y Siavelis no ayuda a entender la lógica de asignación de cargos para subcampeones de la Alianza en 2009. De hecho, ninguna de las variables es estadísticamente significativa.

Ahora bien, un 75,8\% de los subcampeones RN fueron premiados con seguros contra la derrota, mientras un $73,7 \%$ de los subcampeones UDI recibieron igual recompensa. El promedio para toda la Alianza es menor, lo que indica que los independientes y militantes de partidos minoritarios en la Alianza (como Chile Primero) tuvieron menos acceso a premios de consuelo. Por eso, el Modelo 2 añade las variables "DummyUDI" y "DummyRN" para identificar el partido por el que compitió el subcampeón. Este modelo explica el 73\% de los casos, mejorando marginalmente la predicción base de un 66,7\%. Aun así, ninguna variable tiene un efecto estadísticamente significativo para predecir la asignación de premios de consuelo. La militancia en RN o UDI no altera la posibilidad de acceder a premios de consuelo en la forma de cargos de confianza en el Ejecutivo.

El Modelo 3 agrega la variable "Aporte de votos" (el porcentaje de la votación nacional del partido con que contribuye cada subcampeón) para los candidatos 
RN y UDI. Debido a que los partidos también utilizan su votación nacional para evaluar su desempeño, aquellos subcampeones que contribuyen con una alta cantidad de sufragios a la votación nacional del partido pudieran tener mejores argumentos para recibir un premio de consuelo. Aquí excluimos a los candidatos de Chile Primero e independientes porque, al ser tan pocos, cada uno aporta un porcentaje alto a la votación nacional del partido. Aun así, en este modelo ninguna variable resulta significativa. Peor aún, el porcentaje correctamente predicho es de solo $69,8 \%$.

El Modelo 4 incluye la variable "Gasto", como diferencia en el gasto electoral del subcampeón de 2005 y el del subcampeón de 2009 en el distrito respectivo (controlando por inflación). Este modelo contiene 55 casos - excluimos a los senadores, ya que las circunscripciones que fueron a elección en 2005 no fueron a re-elección en 2009. Como los datos de gasto disponibles solo existen a partir de 2004, tampoco pudimos comparar con la elección anterior en 2001 en estas circunscripciones. Por lo tanto, este modelo también excluye la variable Cámara. Este modelo solo predice correctamente el 69,1\% de los casos, y ninguna variable es significativa.

Por último, el Modelo 5 integra la variable sexo. Nuevamente, ninguna variable es significativa. El modelo solo predice con éxito el 68,3\% de los casos, mejorando marginalmente la capacidad de predicción de la afirmación que todos los subcampeones reciben premios de consuelo.

Los modelos del cuadro 5 muestran que la capacidad predictiva de los argumentos presentados por Carey y Siavelis para dar cuenta de la asignación de cargos a los subcampeones de la Concertación entre 1989 y 1997 no tiene la misma fuerza para dar cuenta de la asignación de premios de consuelo a los subcampeones de la Alianza después de 2009. Los datos de Carey y Siavelis son más limitados, probablemente por el menor acceso disponible a datos sobre funcionarios a contrata y honorarios en el sector público en el periodo. Ahora tenemos más información para saber qué ocurre con los subcampeones de la coalición ganadora en el gobierno inmediatamente posterior y podemos evaluar su probabilidad de recibir cargos, aun cuando la capacidad explicativa de los modelos no sea estadísticamente significativa. 
Cuadro 5. Modelos estadísticos en base al modelo Carey y Siavelis (2003) y variables de control

\begin{tabular}{|c|c|c|c|c|c|}
\hline Variables & Modelo 1 & Modelo 2 & Modelo 3 & Modelo $4^{* * *}$ & Modelo 5 \\
\hline Razón Coalición & $\begin{array}{r}0,247 \\
(1,130)\end{array}$ & $\begin{array}{r}0,230 \\
(1,164)\end{array}$ & $\begin{array}{r}0,607 \\
(1,433)\end{array}$ & $\begin{array}{r}0,854 \\
(1,254)\end{array}$ & $\begin{array}{r}-0,046 \\
(1,188)\end{array}$ \\
\hline Razón Alianza & $\begin{array}{r}-0,174 \\
(0,112)\end{array}$ & $\begin{array}{r}-0,114 \\
(0,113)\end{array}$ & $\begin{array}{l}-0,130 \\
(0,210)\end{array}$ & $\begin{array}{r}-0,018 \\
(0,139)\end{array}$ & $\begin{array}{r}-0,164 \\
(0,113)\end{array}$ \\
\hline Cámara & $\begin{array}{r}-0,968 \\
(0,876) \\
\end{array}$ & $\begin{array}{r}-0,862 \\
(0,900)\end{array}$ & $\begin{array}{r}-1,462 \\
(1,555)\end{array}$ & - & $\begin{array}{r}-1,102 \\
(0,904)\end{array}$ \\
\hline Dummy RN & - & $\begin{array}{r}1,230 \\
(0,813)\end{array}$ & - & - & - \\
\hline Dummy UDI & & $\begin{array}{r}1,054 \\
(1,614)\end{array}$ & - & - & - \\
\hline Aporte de votos & - & - & $\begin{array}{r}0,000 \\
(0,000)\end{array}$ & - & - \\
\hline Gasto & - & - & - & $\begin{array}{r}0,594 \\
(0,317)\end{array}$ & - \\
\hline Sexo & - & - & - & - & $\begin{array}{r}0,866 \\
(0,935)\end{array}$ \\
\hline Constante & $\begin{array}{r}2,092 \\
(1,403)\end{array}$ & $\begin{array}{r}0,870 \\
(1,614)\end{array}$ & $\begin{array}{r}2,259 \\
(1,809)\end{array}$ & $\begin{array}{r}-0,844 \\
(1,484)\end{array}$ & $\begin{array}{r}2,385 \\
(1,445)\end{array}$ \\
\hline -2 Log likelihood & 73,847 & 71,492 & 56,089 & 61,021 & 72,900 \\
\hline R de Cox y Snell & 0,096 & 0,129 & 0,083 & 0,092 & 0,109 \\
\hline R Nagelkerke & 0,133 & 0,179 & 0,120 & 0,131 & 0,152 \\
\hline $\mathrm{N}$ & 63 & 63 & 52 & 55 & 63 \\
\hline $\begin{array}{l}\text { \% correctamente } \\
\text { predicho }\end{array}$ & 69,8 & 68,3 & 75,0 & 69,1 & 69,8 \\
\hline
\end{tabular}

* significativo al 0,05 ** significativo al 0,01

*** Para el análisis de la variable "Gasto" se toman solo los casos de diputados

Fuente: elaboración propia

\section{CONCLUSIONES}

Hay motivaciones contrapuestas entre candidatos que buscan conseguir un escaño legislativo y las coaliciones que quieren presentar duplos de candidatos fuertes. Mientras los candidatos prefieren compañeros de lista débiles, las coaliciones aspiran a tener dos postulantes atractivos que aseguren un escaño o incluso consigan un doblaje. El sistema electoral, los mecanismos de selección de candidatos, la ambición política y la incertidumbre sobre los resultados generan escenarios adversos para los aspirantes o candidatos con menores expectativas de éxito.

La política de premios de consuelo en las elecciones de 2009, en forma de cargos de confianza en el Ejecutivo, incentivó la participación de candidatos en la Alianza que tenían pocas posibilidades de conseguir un escaño. Cuando 
la Alianza fue favorita para ganar la elección presidencial, presentó duplos de candidatos más competitivos que la Concertación.

De 63 subcampeones de la Alianza en 2009, 43 recibieron premios de consuelo entregados entre 2010 y 2014. Estos cargos promediaron un sueldo mensual de \$3.938.602 (US\$ 5.931 a tasa de cambio de abril de 2017), subrayando lo atractivo que resultan estos premios como incentivo a la participación de candidatos fuertes.

Utilizamos el modelo de regresión propuesto por Carey y Siavelis para ver si los cargos de confianza se distribuyeron de manera similar a la que utilizó la Concertación entre 1989 y 1997. Además, aplicamos otras variables de control como el partido del candidato, el aporte de votos para el partido a nivel nacional, el gasto realizado en campaña y el sexo del candidato.

El modelo de Carey y Siavelis no tiene buena capacidad explicativa para la Alianza en 2009. Las condiciones bajo las que antes se entregaron los seguros para los subcampeones en la Concertación no son las mismas que se usaron para los de la Alianza después de 2009. Además, los modelos alternativos con otras variables de control tampoco explican significativamente la distribución de los premios de consuelo entre 2010 y 2014.

Ante la incapacidad de los modelos para explicar la entrega de premios de consuelo en la Alianza después de 2009, adelantamos dos respuestas alternativas. La gran cantidad de nombramientos que debe hacer un nuevo gobierno cuando hay alternancia política permite ofrecer cargos de confianza a gran parte de los subcampeones, independientemente de su desempeño electoral. Segundo, debido a la fuerte conexión de la derecha chilena con el mundo empresarial, algunos subcampeones de buen desempeño electoral podrían haber optado por trabajo en el sector privado. Ya que es difícil acceder a información de empleo en el sector privado, no podemos evaluar esa hipótesis. Trabajos posteriores deberán considerar estas explicaciones para entender las dinámicas de distribución de los premios de consuelos para la Alianza.

Aunque las dinámicas de conformación de duplos fuertes de candidatos en Chile ha sido estudiada desde 1989 hasta 2009, cada futura elección presidencial y legislativa ofrecerá una oportunidad para entender mejor las lógicas que explican tanto la decisión de candidatos de presentarse a elecciones donde tienen pocas posibilidades de ganar, como las razones de por qué algunos subcampeones de la coalición ganadora son nombrados a puestos de confianza del Ejecutivo en el periodo inmediatamente posterior. 


\section{REFERENCIAS}

Alarcón, Víctor y Flavia Freidenberg. 2007. “El proceso de selección del candidato presidencial del PAN en 2005". Revista Mexicana de Sociología 69(4): 729-770.

Alcántara, Manuel y Flavia Freidenberg. 2009. Selección de candidatos, política partidista y rendimiento democrático. México: Tribunal Electoral del Distrito Federal, Universidad Nacional Autónoma de México e Instituto de Iberoamérica.

Alcorta, Francisco. 2011. “Carreras políticas una propuesta teórica-metodológica para su análisis". Tesis de licenciatura. Santiago: Universidad Diego Portales.

Black, Gordon S. 1972. "A Theory of Political Ambition: Career Choices and the Role of Structural Incentives". American Political Science Review 66(1): 144-159.

Bunker, Kenneth y Patricio Navia. 2009. "Duración de las carreras de alcaldes 1992-2008". En El Genoma Electoral Chileno, editado por Renato Briceño, Mauricio Morales y Patricio Navia. Santiago: Ediciones Universidad Diego Portales, 261-274.

Carey, John. 2009. "Ingeniería electoral: ¿Qué nos muestran las investigaciones académicas sobre los efectos anticipados de las reformas electorales?". En Reforma del sistema electoral chileno, editado por Arturo Fontaine, Cristián Larroulet, Jorge Navarrete e Ignacio Walker. Santiago: PNUD, CEP, Libertad y Desarrollo, Proyectamérica y Cieplan, 233244.

Carey, John y Matthew Shugart. 1995. "Incentives to Cultivate a Personal Vote: A Rank Ordering of Electoral Formulas". Electoral Studies 14(4): 417-439.

Carey, John y Peter Siavelis. 2003. "El seguro para los subcampeones electorales y la sobrevivencia de la Concertación". Estudios Públicos 90: 5-27.

Cox, Gary. 1999. "Electoral rules and electoral coordination". Annual Review of Political Science 2(1): 145-161.

De Luca, Miguel, Mark P. Jones y María Inés Tula. 2002. “Back Rooms or Ballot Boxes? Candidate Nomination in Argentina". Comparative Political Studies 35(4): 413-36.

Diermeier, Daniel, Michael Keane y Antonio Merlo. 2005. "A Political Economy Model of Congressional Careers," American Economic Review 95(1): 347-373.

Downs, Anthony. 1957. An Economic Theory of Democracy. Boston: Adison Wesley.

Edwards, Santiago. 2009. "Jugando a perdedor: Candidatos con improbables posibilidades de triunfo en elecciones parlamentarias en Chile en 2005". En El Genoma Electoral Chileno, editado por Renato Briceño, Mauricio Morales y Patricio Navia. Santiago: Ediciones Universidad Diego Portales, 297-316.

Fields, Bonnie y Peter Siavelis. 2008. "Procedimientos de selección de candidatos en las democracias nacientes". En Selección de candidatos, política partidista y rendimiento democrático, editado por Manuel Alcántara Sáez y Flavia Freidenberg. México, DF: Tribunal Electoral del Distrito Federal, Universidad Nacional de México e Instituto de Iberoamérica, 61-84.

Fields, Bonnie y Peter Siavelis. 2011. “Endogenizing Legislative Candidate Selection Procedures in Nascent Democracies. Evidence from Spain and Chile". Democratization 18(3), 797-822.

Freidenberg, Flavia. 2003. Selección de candidatos y democracia interna en los partidos de América Latina. Lima: International IDEA y Asociación Civil Transparencia.

Garrido, Carolina. 2009. "Subcampeones de la Concertación: la misión parlamentaria y el retorno a la labor gubernamental". Revista de Ciencia Política 29(1): 111-125.

Garrido, Carolina y Patricio Navia. 2005. “Candidatos fuertes en la Concertación: ¿Seguro para subcampeones o prevalencia de los dos tercios?". Estudios Públicos 99: 165-194.

Groseclose, Tim. 2001. "A Model of Candidate Location when one Candidate has Advantage". American Journal of Political Science 45(4): 862-886.

Hazan, Reuven Y. y Gideon Rahat. 2006. "The Influence of Candidate Selection Methods on Legislatures and Legislators. Theoretical Propositions, Methodological Suggestions and Empirical Evidence". The Journal of Legislative Studies 12(3-4): 366-385. 
Lobos, Manuel, Marcelo Estrella y Christian Parada. 2015. “Evolución histórica de la derecha en Chile desde el retorno a la democracia". En Academia Balmaceda, editado por Manuel Lobos. Santiago: Fundación Balmaceda, 13-83.

Munita, Roberto y Constanza Gillmore. 2009. “Elecciones parlamentarias 2009: Efectos y consecuencias". Santiago: Instituto Libertad.

Navia, Patricio. 2005. "La transformación de votos en escaños: Leyes electorales en Chile, 1833-2003". Política y Gobierno 12(2): 233-276.

Navia, Patricio. 2008. "Legislative Candidate Selection in Chile". En Pathways to Power: Political Recruitment and Candidate Selection in Latin America, editado por Peter Siavelis y Scott Morgenstern. University Park, PA: Penn State Press.

Nohlen, Dieter. 2003. El contexto hace la diferencia: reformas institucionales y el enfoque histórico-empírico. México : Universidad Nacional Autónoma de México, Instituto de Investigaciones Jurídicas : Tribunal Electoral del Poder Judicial de la Federación.

Nohlen, Dieter. 2004. "Sistemas electorales y reforma electoral. Una introducción". Lima, Perú: International IDEA y Asociación Civil Transparencia.

Reynolds, Andrew, Ben Reilly y Andrew Ellis (Eds.). 2006. Diseño de sistemas electorales: el nuevo manual de IDEA Internacional. Estocolmo, Suecia: Internacional IDEA.

Siavelis, Peter. 2005. "La Lógica oculta de la selección de candidatos en las elecciones legislativas chilenas". Estudios Públicos 98: 189-225.

Siavelis, Peter y Scott Morgenstern. 2008. Political Recruitment and Candidate Selection in Latin America. A Framework for Analysis. University Park, PA: Pennsylvania State University Press.

Toro, Sergio y Daniela García. 2008. "Mecanismos de selección de candidatos para el poder legislativo: un examen a las lógicas de mayor y menor inclusión”. En Reforma de los partidos políticos en Chile, eds. Arturo Fontaine, Cristián Larroulet, Jorge Navarrete e Ignacio Walker. Santiago: PNUD, CEP, Libertad y Desarrollo, Proyectamérica y Cieplan, 395-412.

Von Baer, Ena. 2009. "Sistema binominal. Consensos y disensos". En Reforma al sistema electoral chileno, eds. Arturo Fontaine, Cristián Larroulet, Jorge Navarrete e Ignacio Walker. Santiago: PNUD, CEP, Libertad y Desarrollo, Proyectamérica y Cieplan, 177-206.

Patricio Navia es profesor titular de Ciencia Política en la Universidad Diego Portales y de estudios liberales en New York University. Doctorado en New York University, ha publicado artículos sobre el sistema electoral y patrones de votación en Chile. Correo electrónico patricio.navia@nyu.edu

Steffan Sternberg es cientista político de la Universidad Diego Portales, Minor en Ciencias Sociales y diplomado en Pensamiento Contemporáneo por el Instituto de Humanidades de la misma casa de estudios. Correo electrónico steffan.sternberg@gmail.com 